\title{
Residual effect on the robustness of multiqubit entanglement*
}

\author{
Bao-Kui Zhao ${ }^{1,2}$ and Fu-Guo Deng ${ }^{1 \dagger}$ \\ ${ }^{1}$ Department of Physics, Applied Optics Beijing Area Major Laboratory, \\ Beijing Normal University, Beijing 100875, China \\ ${ }^{2}$ College of Nuclear Science and Technology, Beijing Normal University, Beijing 100875, China
}

(Dated: November 19, 2018)

\begin{abstract}
We investigate the relation between the entanglement and the robustness of a multipartite system to a depolarization noise. We find that the robustness of a two-qubit system in an arbitrary pure state depends completely on its entanglement. However, this is not always true in a three-qubit system. There is a residual effect on the robustness of a three-qubit system in an arbitrary superposition of Greenberger-Horne-Zeilinger state and W state. Its entanglement determines the trend of its robustness. However, there is a splitting on its robustness under the same entanglement. Its robustness not only has the same periodicity as its three-tangle but also alters with its three-tangle synchronously. There is also a splitting on the robustness of an $n$-qubit $(n>3)$ system although it is more complicated.
\end{abstract}

PACS numbers: 03.67.Mn, 03.65.Ud, 03.65.Yz

Entanglement is the most nonclassical feature of quantum mechanics and it plays an important role in quantum computation and communication [1]. However, an entangled quantum system is inevitably immersed in an environment and interacts with it in some way, which usually degrades the entanglement of the system. This decoherence introduces some disadvantages on multiqubit entanglement creation and manipulation in quantum information processing. Recently, some works showed that entanglement sudden death (ESD) [2, 3], a peculiar dynamical feature of entangled states, may takes place in an entangled system. In detail, the entanglement of an entangled system may disappear at a finite time although the constituent parts of an entangled state decay asymptotically in time. This interesting phenomenon has been observed in a two-qubit optical system [4, 5].

Recently, some groups have studied the robustness of mulitqubit quantum systems. For example, in 1999, Vidal and Tarrach [6] investigated the robustness of twoqubit systems by considering the minimal amount of mixing with locally prepared states which washes out all entanglement. In 2002, Simon and Kempe 7] showed that the robustness of Greenberger-Horne-Zeilinger (GHZ) entanglement increases with the number of qubits, under local decoherence, modeled by partially depolarizing channels acting independently on each qubit. They also pointed out that the amount of entanglement of a multiqubit system and its robustness do not have a simple relation. In 2008, Aolita et al. [8] showed clearly that the time at which such entanglement of a pure GHZ-state quantum system becomes arbitrarily small is inversely proportional to $N$, although its ESD time increases with $N$. In 2008, Batle and Casas [9] found that the entanglement in a three-qubit system with the measure of Mer-

\footnotetext{
*published in Phys. Rev. A 82, 014301 (2010)

${ }^{\dagger}$ Corresponding author: fgdeng@bnu.edu.cn.
}

min's inequality [10] and its robustness contain similarities, but do not have a simple relation. In 2009, Liu and Fan 11] investigated the decay of entanglement of a generalized $N$-qudit GHZ state under local decoherence and obtained results similar to those reported in Refs. [7, [8]. In 2009, Borras et al. [12] investigated the decay of the amount of entanglement of a multqubit system experiencing a decoherence process.

In this article, we investigate the relation between the entanglement and the robustness of a multipartite system to a depolarization noise. For a two-qubit system in an arbitrary pure entangled state, its robustness increases synchronously with its entanglement. For a three-qubit system, this phenomenon, however, disappears and the robustness of GHZ-like states, which have only threetangle (the entanglement shared by all the three qubits) without concurrence, is the upper boundary of symmetrical three-qubit pure states. It is interesting to point out that there is a residual effect on the robustness of a threequbit system in an arbitrary superposition of a GHZ state and a $\mathrm{W}$ state. Its entanglement, on the one hand, determines the trend of its robustness. On the other hand, there is a splitting on its robustness. Its robustness not only has the same periodicity as its three-tangle but also alters with its three-tangle synchronously. This interesting phenomenon takes place in a four-qubit system and a five-qubit system, but there is not a good measure on their entanglements shared by all the qubits in this time.

In the present study, each qubit in multiqubit entangled quantum systems is coupled to its own environment individually. That is, our study is under local decoherence, modeled by partially depolarizing channels acting independently on each qubit, the same as that in Ref. [7]. The dynamics of each qubit in a three-particle entangled quantum system is governed by a master equation from which one can obtain a completely positive tracepreserving map $\varepsilon$ which describes the corresponding evolution [12]: $\rho_{i}(t)=\varepsilon(t) \rho_{i}(0)$. In the Born-Markovian approximation the maps (or channels) can be described 
using its Kraus representation [12, 13], that is,

$$
\varepsilon\left(\rho_{i}(0)\right)=\sum_{j=1}^{3} E_{j i}(t) \rho_{i}(0) E_{j i}^{\dagger}(t),
$$

where $E_{j}(t)(j=1,2,3)$ are the so-called Kraus operators needed to completely characterize the channel. In detail, the partially depolarizing channel $C_{d}$ for each qubit is defined by applying the completely depolarizing channel with a probability $d$, and applying the identity transformation with a probability $1-d$. This corresponds to the following transformation [7]

$$
\begin{aligned}
|i\rangle\langle j| & \longrightarrow(1-d)|i\rangle\langle j|+d \delta_{i j} \frac{1}{2} \mathbf{1} \\
P_{k} & \longrightarrow \frac{1+s}{2} P_{k}+\frac{1-s}{2} P_{k \oplus 1} \\
\sigma_{+} & \longrightarrow s \sigma_{+} \\
\sigma_{-} & \longrightarrow s \sigma_{-} .
\end{aligned}
$$

Here $s=1-d, k \in\{0,1\}$, and $k \oplus 1$ means that the sum of $k$ and $1 \bmod 2$. $P_{0}=|0\rangle\left\langle 0\left|, P_{0}=\right| 1\right\rangle\left\langle 1\left|, \sigma_{+}=\right| 0\right\rangle\langle 1|$, and $\sigma_{-}=|1\rangle\langle 0|$.

First we explore the connection between the entanglement of a two-qubit quantum system and its robustness and then generalize it in a three-qubit system.

Under the depolarized noise $C_{d}$ shown in $\mathrm{Eq}(2)$ on each qubit, the robustness of a given $n$-party entangled state $\rho$ is defined as the critical amount of depolarization $d_{\text {crit }}$ where $C_{d}^{\otimes n}(\rho)$ becomes separable, or- in the absence of a necessary and sufficient condition- ceases to fulfill certain sufficient conditions for entanglement 7, 14-16]. The entanglement (negativity- $N$ ) of a quantum system in an arbitrary pure state can be obtained with the method in Refs. 17-19] and its robustness $\left(R=d_{\text {crit }}\right)$ can be calculated with the PPT criterion [14]. Negativity of a bipartite quantum system is defined as $N=\Sigma|\lambda|$, where $\lambda$ are the negative eigenvalues of $\rho^{T_{B}}$ and $T_{B}$ denotes the partial transpose with respect to the subsystem $B$. For an arbitrary bipartite pure state, its entanglement is completely determined by its Schmidt coefficients, and the depolarizing channel is basis independent. With a numerical simulation, we give the relation between the entanglement and the robustness in two-qubit pure states in Fig,1. It is explicit that the more the entanglement of a two-qubit entangled pure state, the more its robustness. That is, the two systems with the same entanglement have the same robustness although they are in two different entangled pure states. Also, we find that there is a simple analytical expression for the relation between $N$ and $R$ of a two-qubit system in an arbitrary pure state, that is,

$$
R=1-\frac{1}{\sqrt{1+2 N}}
$$

That is, its robustness depends completely on its entanglement.

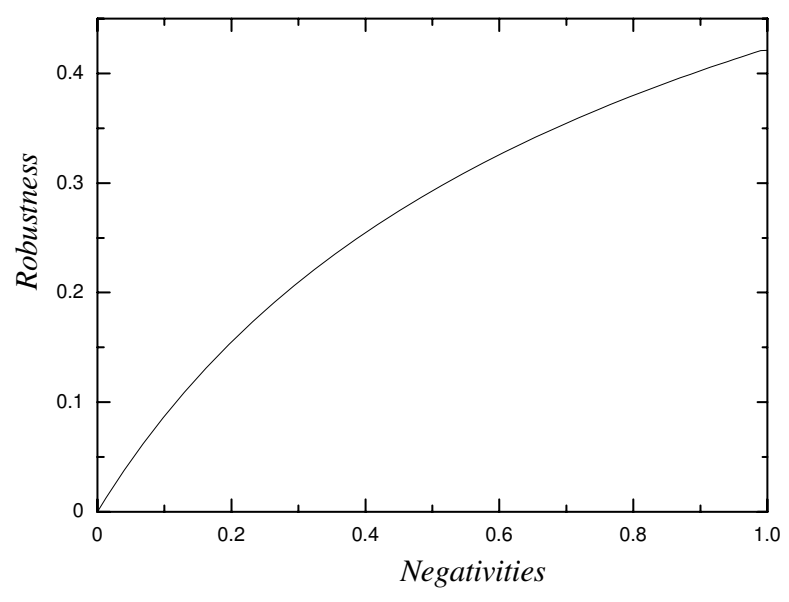

FIG. 1: The relation between the robustness and the negativity of a two-qubit system in a pure entangled state.

For a three-qubit system, there are two inequivalent classes of genuine tripartite entanglement 20], represented by the GHZ state $|G H Z\rangle=\frac{1}{\sqrt{2}}(|000\rangle+|111\rangle)$ and the $\mathrm{W}$ state $|W\rangle=\frac{1}{\sqrt{3}}(|001\rangle+|010\rangle+|100\rangle)$. Each threequbit pure state can be converted into either a GHZ-class state or a W-class state by stochastic local quantum operations assisted by classical communication (SLOCC) [20, 21]. Our question is, whether or not a three-qubit quantum system has the same result as a two-qubit quantum system? That is, is there the explicit phenomenon that the more the entanglement of a three-qubit entangled pure state, the more its robustness?

As the depolarizing channel is symmetric to each qubit in a three-qubit system, we consider two classes of symmetric pure states, which are invariable under the permutations of the three particles, to study the relation between their robustness and their entanglements, i.e., the GHZ-like state $|\Lambda\rangle$ and the W-like state $|\Omega\rangle$,

$$
\begin{aligned}
|\Lambda\rangle_{A B C} & =\sqrt{a}|000\rangle+\sqrt{1-a}|111\rangle, \\
|\Omega\rangle_{A B C} & =\sqrt{b}|000\rangle+\sqrt{\frac{1-b}{3}}(|001\rangle+|010\rangle+|100\rangle),
\end{aligned}
$$

where $a, b \in[0,1]$. The relations between the entanglements and the robustness of the states $|\Lambda\rangle$ and $|\Omega\rangle$ are plotted with the solid (red ) line and the dashed (magenta) line, respectively, in Fig 2 For each class of entangled states, on the one hand, the more the entanglement, the more its robustness. On the other hand, the GHZ-like state $|\Lambda\rangle_{A B C}$ is more robust than the W-like state $|\Omega\rangle_{A B C}$ with the same entanglement under the depolarizing channel $C_{d}^{\otimes 3}$.

More generally, an arbitrary symmetrical three-qubit entangled pure state can be written as

$$
|\Psi\rangle_{A B C}=c_{1}|000\rangle+c_{2} \frac{1}{\sqrt{3}}\{|001\rangle+|010\rangle+|100\rangle\}
$$




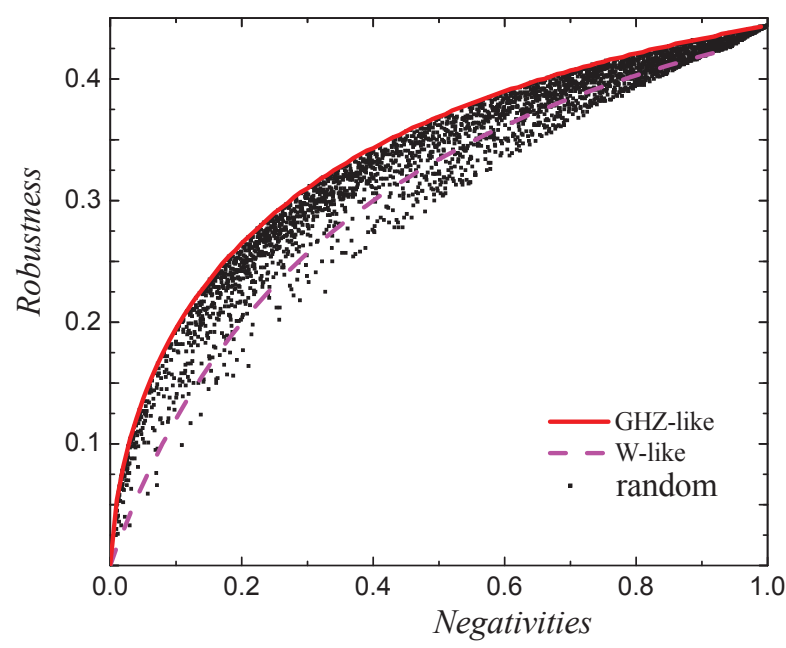

FIG. 2: (Color online) The relation between the robustness and the negativity of a three-qubit system in a GHZ-like state, a W-like state, and a symmetrical three-qubit pure state is shown with a solid (red) line, a dashed (magenta) line, or a solid (black) dot, respectively. For a GHZ-like state, negativity $\in[0,1]$. For a W-like state, negativity $\in\left[0, \frac{2 \sqrt{2}}{3}\right]$.

$$
+c_{3} \frac{1}{\sqrt{3}}\{|110\rangle+|101\rangle+|011\rangle\}+c_{4}|111\rangle
$$

where $c_{1}, c_{2}, c_{3}$, and $c_{4}$ are four complex numbers and satisfy $\left|c_{1}\right|^{2}+\left|c_{2}\right|^{2}+\left|c_{3}\right|^{2}+\left|c_{4}\right|^{2}=1$. We choose randomly a great number of symmetrical three-qubit entangled pure states with the Haar measure 22] and calculate their negativity. With a numerical simulation, we give the relation between the entanglement and the robustness in symmetrical three-qubit pure states $|\Psi\rangle_{A B C}$ in Fig 2 with solid black dots. Different from two-qubit states, the robustness of a symmetrical three-qubit state does not only depend on its negativity. Generally speaking, the more the entanglement, the more the robustness of a symmetrical three-qubit pure state. However, one can see that there is a subset of states which have the same entanglement but different robustness. Moreover, the GHZ-like state is more robust than others under the same entanglement. That is, the robustness of GHZ-like states, which have only three-tangle 23 25] (it is called originally the residual entanglement [23]) without concurrence, is the upper boundary of symmetrical three-qubit pure states, but W-like states without three-tangle are not the most fragile ones. This phenomenon implies that the three-tangle is the highest-quality (the most robust) entanglement against the depolarization noise.

In a three-qubit system, the negativity only measures a bipartite entanglement, while the three-tangle quantifies genuine multipartite correlations. In order to explore the role of different entanglement components (three-tangle or negativity) in robustness of a three-qubit system, we should study the robustness of a class of three-qubit pure states which have the same entanglement and different robustness. Fortunately, we find that the superpositions of the GHZ state and the W state have different robustness under the same entanglement (negativity), that is,

$$
|Z(a, \varphi)\rangle=\sqrt{a}|G H Z\rangle-e^{i \varphi} \sqrt{1-a}|W\rangle .
$$

The parameter $a \in[0,1]$ is used to identify the proportion of the GHZ state and $\varphi$ is used to represent the relative phase between the GHZ state and the W state. The negativities of these three-qubit systems can be described as

$$
N[|Z(a, \varphi)\rangle]=\frac{\sqrt{5 a^{2}-4 a+8}}{3} .
$$

It is obvious that the entanglement of the state $|Z(a, \varphi)\rangle$ does not depend on the phase factor $\varphi$. The relation between its entanglement and the coefficient $a$ is shown in the left inset in Fig. 3 However, its robustness depends on not only the coefficient $a$ but also the phase factor $\varphi$, shown in Fig 3 for the cases $\varphi=\pi / 3,2 \pi / 9,2 \pi / 15, \pi / 15,0$. Moreover, its robustness $R[|Z(a, \varphi)\rangle]$ varies periodically with the phase $\varphi$; that is,

$$
R[|Z(a, \varphi)\rangle]=R[|Z(a, \varphi+2 \pi / 3)\rangle] .
$$

The periods of the robustness $T=2 \pi / 3$. In each period, $R[|Z(a, \pi / 3-\Delta \varphi+n T)\rangle]=R[|Z(a, \pi / 3+\Delta \varphi+n T)\rangle]$,

where $\Delta \varphi \in[0, \pi / 3]$ and $n=0,1,2, \ldots$

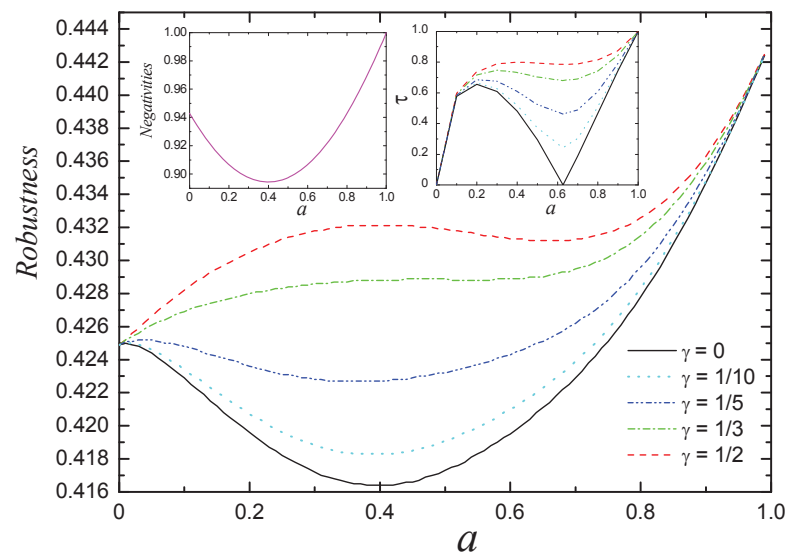

FIG. 3: (Color online) Robustness of the state $|Z(a, \varphi)\rangle=$ $\sqrt{a}|G H Z\rangle-e^{i \varphi} \sqrt{1-a}|W\rangle$ versus the coefficient $a$ with different phase facotrs $\varphi=\gamma \cdot \frac{2 \pi}{3}$ (rad) (from top to bottom: $\gamma=1 / 2,1 / 3,1 / 5,1 / 10,0)$. The left inset shows the relation between the entanglement of the state $|Z(a, \varphi)\rangle$ and the coefficient $a$. The right inset shows the residual entanglement of the state $|Z(a, \varphi)\rangle$ versus the coefficient $a$ with different phase factors $\varphi$.

With a given coefficient $a$, which means that the states $|Z(a, \varphi)\rangle$ have the same components of the GHZ state and the W state, the larger the phase $\varphi$, the greater the 
robustness of the state $|Z(a, \varphi)\rangle$ in each period. With a given phase factor $\varphi$, the robustness of the state $|Z(a, \varphi)\rangle$ appears to have two different effects. When $\varphi$ is small, a tripartite quantum system in the state $|Z(a, \varphi)\rangle$ appears to have a normal robustness effect in which its robustness alters synchronously with its negativity. That is, its robustness decreases with the coefficient $a$ when $a \leq 0.4$ and increases with $a$ when $a>0.4$, same as its negativity. However, an abnormal robustness effect takes place when $\varphi$ becomes large. Specifically, the robustness of the state $|Z(a, \varphi)\rangle$ alters inversely with its negativity when $a$ is not large. In principle, it is explicit that there is a splitting on the robustness of the state $|Z(a, \varphi)\rangle$, which is completely different from its negativity. The fluctuation of the robustness is at most $4 \%$. That is, the negativity of a three-qubit system determines the trend of its robustness. Also, there is a residual effect on the robustness although the fluctuation is small.

It is interesting to point out that the feature of the robustness of the state $|Z(a, \varphi)\rangle$ agrees with that of its three-tangle. For the state $|Z(a, \varphi)\rangle$, its three-tangle can be calculated as [25]

$$
\tau[|Z(a, \varphi)\rangle]=\left|a^{2}-\frac{8 \sqrt{6}}{9} \sqrt{a(1-a)^{3}} e^{3 i \varphi}\right| .
$$

It is periodic in the phase $\varphi$ with the same periods of $T_{\tau}=T=2 \pi / 3$ as the robustness of the sate $|Z(a, \varphi)\rangle$, shown in the right insert in Fig 3 for the cases $\varphi=$ $\pi / 3,2 \pi / 9,2 \pi / 15, \pi / 15,0$. Also, in each period,

$\tau[|Z(a, \pi / 3-\Delta \varphi+n T)\rangle]=\tau[|Z(a, \pi / 3+\Delta \varphi+n T)\rangle]$.

In order to show explicitly the effect of the three-tangle on the the robustness of the state $|Z(a, \varphi)\rangle$, we give the relation between the robustness $R$ and the phase $\varphi$ and that between $\tau$ and the phase $\varphi$ under a given coefficient $a=0.2,0.4,0.6$, shown in Fig, For a state $|Z(a, \varphi)\rangle$ under a given coefficient $a$, both its robustness and its three-tangle $\tau$ increase with the phase $\varphi$ when $0 \leq \varphi \leq$ $\pi / 3$ and decrease with the phase $\varphi$ when $\pi / 3 \leq \varphi \leq$ $2 \pi / 3$. That is, the robustness and the three-tangle have the same monotonicity.

For a four-qubit system and a five-qubit system in the state $|Z(a, \varphi)\rangle_{n}=\sqrt{a}|G H Z\rangle_{n}-e^{i \varphi} \sqrt{1-a}|W\rangle_{n}$ $(n=4,5)$, there is also the phenomenon as a three-qubit system. That is, there is a splitting on its robustness. Here $|G H Z\rangle_{n}=\frac{1}{\sqrt{2}}(|00 \cdots 0\rangle+|11 \cdots 1\rangle)$ and $|W\rangle_{n}=$ $\frac{1}{\sqrt{n}}(|0 \cdots 01\rangle+|0 \cdots 10\rangle+\cdots+|1 \cdots 00\rangle)$. The robustness has the periods $T_{R_{n}}=2 \pi / n$, i.e., $R_{n}\left[|Z(a, \varphi)\rangle_{n}\right]=$ $R_{n}\left[|Z(a, \varphi+2 \pi / n)\rangle_{n}\right]$. Unfortunately, there is lack of studies on $n$-tangle ( $n \geq 4$, i.e., the entanglement shared by all the $n$ qubits). For two-qubit systems, their entanglements are just the ones shared by both the qubits, and the robustness of each two-qubit pure state depends completely on its negativity.

In summary, we have investigated the relation between the entanglement and the robustness of a multipartite system to a depolarized noise. For a two-qubit system

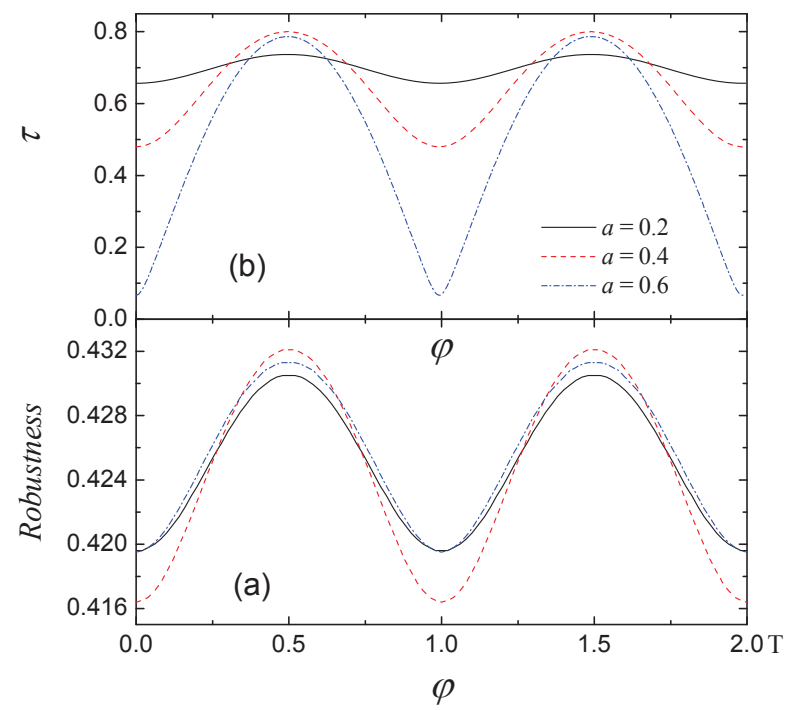

FIG. 4: (Color online) (a) Periodicity and monotonicity of the robustness of the state $|Z(a, \varphi)\rangle=\sqrt{a}|G H Z\rangle-e^{i \varphi} \sqrt{1-a}|W\rangle$ versus $\varphi=\gamma \cdot \frac{2 \pi}{3}(\mathrm{rad})$ with a different coefficient $a$ (from top to bottom: $a=0.2,0.4,0.6)$; (b) Periodicity and monotonicity of the three-tangle of the state $|Z(a, \varphi)\rangle$ versus $\varphi$ with a different coefficient $a$.

in an arbitrary pure entangled state, its negativity determines completely its robustness. For a three-qubit system in a general symmetrical pure state, the trend of its robustness is, on the one hand, determined by its entanglement. The robustness of GHZ-like states, which have only three-tangle without concurrence, is the upper boundary of symmetrical three-qubit pure states, which implies that the three-tangle is the highest-quality entanglement against the depolarization noise. On the other hand, there is a residual effect on the robustness. That is, the robustness of a three-qubit system in an arbitrary superposition of a GHZ state and a W state not only has the same periodicity as its three-tangle but also alters with its three-tangle synchronously. There is also a splitting on the robustness of a four-qubit system and a five-qubit system although there is not a good measure for their $n$-tangle $(n=4,5)$.

One should certainly be careful in drawing general conclusions about the robustness and the entanglement of a multipartite system. Nevertheless, our results show that the three-tangle affects the robustness of a three-qubit system as they have the same periodicity and monotonicity but not the extent. Moreover, the splitting on the robustness of a system with more than three qubits provides an open question for people to study the definition of the entanglement shared by all the qubits. Also, it may give some useful information about decoherence in quantum information processing. On the other hand, are there other factors that affect the robustness of mutli-qubit system? Dose this phenomenon exist in high-dimensional systems or not? These are still some 
open questions of interest to us.

This work is supported by the National Natural Science Foundation of China under Grant No. 10974020, A Foundation for the Author of National Excellent
Doctoral Dissertation of P. R. China under Grant No. 200723, and the Beijing Natural Science Foundation under Grant No. 1082008.
[1] M. A. Nielsen and I. L. Chuang, Quantum Computation and Quantum Information (Cambridge University Press, Cambridge, 2000).

[2] P. J. Dodd and J. J. Halliwell, Phys. Rev. A 69, 052105 (2004).

[3] T. Yu and J. H. Eberly, Phys. Rev. Lett. 93, 140404 (2004); 97, 140403 (2006).

[4] M. P. Almeida, F. de Melo, M. Hor-Meyll, A. Salles, S. P. Walborn, P. H. Souto Ribeiro, and L. Davidovich, Science 316, 579 (2007).

[5] A. Salles, F. de Melo, M. P Almeida, M. Hor-Meyll, S. P. Walborn, P. H. Souto Ribeiro, and L. Davidovich, Phys. Rev. A 78, 022322 (2008).

[6] G. Vidal and R. Tarrach, Phys. Rev. A 59, 141 (1999).

[7] C. Simon and J. Kempe, Phys. Rev. A 65, 052327 (2002).

[8] L. Aolita, R. Chaves, D. Cavalcanti, A. Acin, and L. Davidovich, Phys. Rev. Lett. 100, 080501 (2008).

[9] J. Batle and M. Casas, Int. J. Quantum Inform. 6, 237 (2008).

[10] N. D. Mermin, Phys. Rev. Lett. 65, 1838 (1990).

[11] Z. Liu and H. Fan, Phys. Rev. A 79, 064305 (2009).

[12] A. Borras, A.P. Majtey, A.R. Plastino, M. Casas and A.Plastino, Phys. Rev. A 79, 022108 (2009).

[13] K. Kraus, States, Effect, and Operation: Fundamental Notions in Quantum Theory (Springer-Verlag, Berlin,
1983).

[14] A. Peres, Phys. Rev. Lett. 77, 1413 (1996).

[15] M. Horodecki, P. Horodecki, and R. Horodecki, Phys. Lett. A 223, 1 (1996).

[16] A. Sørensen, L. M. Duan, J. I. Cirac, and P. Zoller, Nature (London) 409, 62 (2001).

[17] K. Życzkowski, P. Horodecki, A. Sanpera, and M. Lewenstein, Phys. Rev. A 58, 883 (1998).

[18] K. Życzkowski, Phys. Rev. A 60, 3496 (1999).

[19] G. Vidal and R. F. Werner, Phys. Rev. A 65, 032314 (2002).

[20] W. Dür, G. Vidal, and J. I. Cirac, Phys. Rev. A 62, 062314 (2000).

[21] C. H. Bennett, S. Popescu, D. Rohrlich, J. A. Smolin, and A. V. Thapliyal, Phys. Rev. A 63, 012307 (2001).

[22] K. Życzkowski and M. Kuś, J. Phys. A: Math. Gen. 27, 4235 (1994).

[23] V. Coffman, J. Kundu, and W. K. Wootters, Phys. Rev. A 61, 052306 (2000).

[24] A. Wong and N. Christensen, Phys. Rev. A 63, 044301 (2001).

[25] R. Lohmayer, A. Osterloh, J. Siewert and A. Uhlmann, Phys. Rev. Lett. 97, 260502 (2006). 